\title{
Redes universitarias regionales de innovación, nuevo escenario de aprendizaje social
}

\author{
Felipe Vega González ${ }^{1}$ \\ Universidad Tecnológica de Pereira (Colombia) \\ fevego@utp.edu.co
}

Recepción: 9/05/2013

Evaluación: 22/10/2013

Aceptación: 13/12/2013

Artículo de Reflexión

DOI:http: / / dx.doi.org / 10.9757 / Rhela.22 / 10

\section{RESUMEN}

El estudio de nuevos escenarios en los que se proyectan las relaciones entre educación y comunicación debe llevarnos a la comprensión de las relaciones entre los distintos actores sociales para generar conocimiento y dar vida a unas nuevas relaciones de enseñanza-aprendizaje hacia la sociedad del conocimiento.

La reconfiguración de la escuela pasa por su vinculación al conjunto de redes de innovación, sobrepasando el terreno de lo técnico-productivo y erigiéndose, dentro de una red de relaciones de creciente complejidad, en la estructuración de una alternativa que involucra estrechamente la gestión y producción del conocimiento con su impacto regional.
Desarrollo de redes por medio de las cuales se está reconfigurando el universo educativo y que constituye la posibilidad de revivir el protagonismo perdido de la universidad en cuanto conciencia crítica de la sociedad hacia la solución de los problemas fundamentales que enfrenta la sociedad en general y las regiones en particular.

Palabras clave: Revista Historia de la EducacionLatinaomericana, comunicación educativa, sociedad del conocimiento, universidad, reconfiguración de la escuela, redes de innovación.

\footnotetext{
1 Candidato a Doctor en Ciencias de la Educación, RUDECOLOMBIA, Economista, Especialista en Gerencia de Tecnología,Magíster en Comunicación Educativa integrante del grupo de investigación: Políticas, sociabilidades y representaciones históricoeducativas.
} 
University regional networks of innovation new social learning scenario

ABSTRACT

The study of new scenarios in which the relationships are projected between the education and communication should lead us to an understanding of the relationships between the different social actors to generate knowledge and give life to the new relationships of teachinglearning towards the knowledge society.

The reconfiguration of the school crosses through its linkage to the set of innovation networks, overcoming the field of technical-productive and, within a network of relations of increasing complexity, in the structuring of an alternative that involves closely the management and production of knowledge with its regional impact.

Development of network through which it is reshaping the educative universe and that has the possibility of reviving the lost prominence of the university as critical conscience of society towards the solution of the fundamental problems facing the society in general and the regions in particular.

Key words: Journal History of the Latin-American Educatio, communicative education, society of knowledge, university, reconfiguration of the school, innovation networks.
Universidade regional de inovação da rede nova etapa de aprendizagem social

\section{RESUMO}

O estudo de novos cenários em que a relação entre a educação eoprojeto de comunicação deve levar à compreensão das relações entre os atores sociais para gerar conhecimento e dar vida a uma nova relação de ensino e aprendizagem para uma sociedade do conhecimento.

A reconfiguração da escola passa por sua ligação com todas as redes de inovação, ultrapassando o domínio do conhecimento técnico- produtiva e estabelecer-se dentro de uma rede de relações de complexidade crescente na estruturação de uma alternativa que envolve perto a gestão e produção do conhecimento com o seu impacto regional.

Desenvolvimento de redes através do qual está a remodelar o universo educacional e que é a possibilidade de reviver o papel perdido da universidade como uma consciência crítica da sociedade para a solução dos problemas fundamentais da sociedade e regiões em particular.

Palavras-chave: Revista História da Educação Latino-americanacomunicação educativa, sociedade do conhecimento, faculdade, escola, reconfiguração de redes de inovação.

\section{INTRODUCCIÓN}

Un presupuesto fundamental para la presentación de las reflexiones y propuestas que aquí se exponen, lo constituye la identificación general de las características y determinantes de la innovación en el contexto contemporáneo; cualquiera que sea su modalidad se genera en el desarrollo de redes, definidas como un nudo de relaciones entre actores que se benefician de pertenecer a ellas. En el caso de la innovación, las redes ayudan a inducir y difundir los cambios tecnológicos y facilitan el traspaso de conocimientos y experiencias acumuladas entre los actores participantes. Redes que emergen y se proyectan por la interacción, con diversos grados y alcances, de los 
distintos actores que constituyen el tejido social, bajo las relaciones predominantes y en un estrecho encadenamiento entre los distintos niveles: local, regional, nacional y transnacional. Bajo este marco de estudio es posible identificar suficientes y muy diversos elementos para sustentar que están dadas las condiciones que caracterizan la innovación en la universidad como un componente misional adicional a los tres que vienen históricamente configurando la misión universitaria: investigación, docencia y extensión y que sobre esta base tiene sentido explicar las redes universitarias regionales de innovación como un nuevo escenario de aprendizaje social.

\section{Globalizaciones y nuevos escenarios productivos y educativos}

El estudio de las nuevas condiciones, las nuevas reglas del juego económico y social, a las puertas de la sociedad y la economía del conocimiento, deben permitirnos una comprensión de la recomposición que han sufrido las formas de dominación y dependencia, en la cadena productiva que interconecta países desarrollados que manejan los hilos de la transnacionalización y países dependientes que ven frustrado el camino al desarrollo. Esto nos lanza el reto de crear, desde el mundo y las regiones atrasadas del mismo, formas de interacción y construcción de conocimiento que configuren innovación en el campo de las relaciones enseñanza-aprendizaje.

El aprendizaje que implica la apropiación social del conocimiento es la base fundamental para la generación de innovación y cambio social.

"El aprendizaje es el proceso por medio del cual el conocimiento crea o fortalece capacidades y habilidades en las personas u organizaciones que se lo apropian, convirtiéndose en dinamizador del cambio en la sociedad, en sus instituciones, o en las empresas de un pais". ${ }^{2}$

Unas nuevas reglas del juego económico y social emergen con toda su proyección en las últimas dos décadas, en un rápido e intenso proceso de cambio dominado por tres tendencias dinámicas y complejas: el desarrollo de la sociedad de la información, la globalización y el progreso científicotecnológico, generando nuevas oportunidades pero de igual manera grandes desafíos. $^{3}$

2 Marco Raúl Jaramillo, Educacion(es) en la(s) globalización(es).Entre el pensamiento ùnico y la nueva crítica.(Bogotá: Edicionesdesdeabajo,2006), 75.

3 Fernando Chaparro Osorio, "De las sociedades de la información a las sociedades del conocimiento", en Chaparro.Conocimiento, 


\section{Hacia la sociedad del conocimiento y nuevos modos de produc- ción del conocimiento}

El concepto de sociedades del conocimiento involucra dimensiones sociales, éticas y políticas mucho más vastas que la noción de sociedad de la información basada en los progresos tecnológicos. Con esta visión en el concepto se deben tener en cuenta los conocimientos locales o autóctonos en las sociedades del conocimiento, cuyos modelos de desarrollo varían considerablemente en relación con las características del conocimiento científico tal como es entendido en occidente. ${ }^{4}$

La importancia que estas nuevas sociedades conceden a los derechos fundamentales se traducirá por una focalización especial en:-la libertad de opinión y expresión (artículo 19 de la Declaración Universal de DerechosHumanos) y la libertad de información, el pluralismo de los media y la libertad académica;- el derecho a la educación y sus corolarios: la gratuidad de la enseñanza básica y la evolución hacia la gratuidad de los demás niveles de enseñanza (artículo 26 de la Declaración Universal de Derechos Humanos y artículo 13 del Pacto Internacional de Derechos Económicos, Sociales y Culturales); el derecho a "tomar parte libremente en la vida cultural de la comunidad, a gozar de las artes y a participar en el progreso científico y en los beneficios que de él resulten (párrafo 1 del artículo 27 de la Declaración Universal de Derechos Humanos). ${ }^{5}$

Los valores y prácticas de creatividad e innovación serán fundamentales para responder mejor a las nuevas necesidades de la sociedad y establecer así procesos de colaboración, frente al modelo de competitividad que ya ha demostrado su inoperancia para la construcción de equidad. La reflexión sobre las sociedades del conocimiento y su edificación permite replantearse el propio concepto de desarrollo.

La causa final del desarrollo humano cimentado sobre la búsqueda de las libertades sustanciales. Estas libertades comprenden las posibilidades elementales de acceso a la educación, el mercado de trabajo, la salud y los productos y la participación en las decisiones políticas, la igualdad de acceso a la información y el derecho a la seguridad colectiva.

innovación y construcción de sociedad, (Bogotá: TercerMundoeditores, 1998).

4 UNESCO, Consejo del Informe Mundial. Hacia las sociedades del conocimiento, (Paris Ediciones Unesco, PDF,2005). Consultado mayo 10 de 2010

5 Op.cit. pág.18. 
América Latina puede cambiar su estructura productiva y sus capacidades sociales, si reconoce su potencial de innovación y esto lo logramos reflejar en un modelo de desarrollo sustancialmente distinto que propicie la interacción entre todos los actores sociales.Si bien, en todas las épocas y culturas el conocimiento ha sido relevante, lo característico de la época contemporánea es el rol central que este adquiere, así como la velocidad con la cual se expande, gracias a los espectaculares avances de la ciencia, su internacionalización y los radicales cambios en la producción y aplicación del conocimiento a todas las esferas de la vida social (económica, cultural, ambiental, políticoinstitucional).

A tal fin, básicamente se requiere de la capacidad para generar conocimiento sobre su realidad y su entorno, y para utilizar dicho conocimiento en el proceso de concebir, forjar y construir su futuro ${ }^{6}$.

La innovación, en cualquiera de sus manifestaciones dentro de la vida social, no ocurre como un suceso aislado, sino en el marco de ciertas redes de actores. La universidad, desde cualquiera de las áreas del conocimiento en las que se relaciona con la sociedad, es una de las fuentes fundamentales desde las cuales se genera la innovación en cualquiera de sus manifestaciones: políticas, económicas, sociales, culturales, ambientales, tecnológicas y organizacionales.

El desarrollo [...] es un proceso multidimensional que en forma paulatina acumula -en un sistema territorial-las condiciones que mediante mecanismos sinápticos y sinérgicos harán que en la dinámica de una emergencia evolutiva en un momento dado emerja un nuevo "estado del sistema" al cual con propiedad se puede denominar como 'desarrollo'?

Es el mundo mismo el contexto de cualquier conocimiento político, económico, antropológico, ecológico. Lo nuevo, dentro del contexto actual y así crecientemente hacia el futuro, debe dar respuesta a la complejidad, unión entre la unidad y la multiplicidad. La educación deberá promover una"inteligencia general" apta para referirse de manera multidimensional, a lo complejo, al contexto en una concepción global.

\footnotetext{
6 Fernando Chaparro Osorio. Conocimiento, innovación y construcción de sociedad. Bogotà: Tercer Mundo Editores, 1998

7 Sergio Boisier.Un ensayo epistemológico y axiológico sobre gestión del desarrollo territorial: conocimiento y valores,(Santiago de Chile:Cepal, 2005).
} 
La creatividad y la innovación se enfrentan al principio de reducción que conduce naturalmente a restringir lo complejo a lo simple: "Aplica a las complejidades vivas y humanas la lógica mecánica y determinista de la maquina artificial. Igualmente, cuando obedece estrictamente al postulado determinista, el principio de reducción oculta el riesgo, la novedad, la invención". ${ }^{8}$

Debemos respondernos, entonces, interrogantes definitivos que nos permitan ubicar con claridad las transformaciones que son necesarias desplegar desde la base misma del sistema educativo. El ser humano es a la vez físico, biológico, psíquico, cultural, social, histórico. Esta condición humana compleja se nos olvidó y debemos recuperarla por medio de una educación integradora.

Debemos apostar por una ciudadanía terrestre, para la cual el siglo XX ha aportado los gérmenes y embriones y la educación del XXI tiene en su nueva misión enseñar sus componentes. Enseñar principios de estrategia que permitan aprender a navegar en un océano de incertidumbres por medio de archipiélagos de certeza. La comprensión es al mismo tiempo medio y fin de la comunicación humana. Ahora bien la educación para la comprensión está ausente de nuestras enseñanzas. El planeta necesita comprensiones mutuas en todos los sentidos.

A lo largo de la historia se nos ha ofrecido constantemente la certidumbre. Accedemos a unaépoca en la cual todo conduce a sustentar nuestras búsquedas bajo la luz de la incertidumbre, "no como una ceguera desafortunada y temporal ni como un obstáculo insuperable al conocimiento, sino mas bien como una increíble oportunidad para imaginar, crear ybuscar". ${ }^{9}$

El pluralismo se convierte en uno de los caminos que nos permitirá incentivar la creatividad y la innovación en la búsqueda de respuestas hacia un mejor universo, que no admite respuestas únicas y unilaterales. Se impone, ante estas realidades y para generar el nuevo escenario de la innovación, la generalización del trabajo en redes del conocimiento. La conformación y desarrollo de redes regionales de innovación al servicio de nuevas condiciones productivas y de existencia se convierte así en reto claro

8 Edgar Morin, “Principios del conocimiento pertinente - Reducción y disyunción”, en Los siete saberes necesarios para la educación del futuro,(Paris-Francia: Unesco,1999).

9 Immanuel Wallerstein, El legado de la sociología, la promesa de la ciencia social, (Monteral:XXIV Congreso Mundial de Sociología,1998). 
hacia el desarrollo de la resistencia activa frente a la hegemonía que detenta el poder económico y social en los ámbitos nacional y planetario.

Esas nuevas condiciones de existencia, atravesada por múltiples y novedosos niveles de interacción entre la comunicación y la educación, ha propiciado la emergencia de un nuevo modo de producción de conocimiento. Las universidades tendrán que desarrollar en este nuevo contexto estructuras que promuevan y premien a los grupos de creatividad. Será necesario el desarrollo de un ethos basado en trabajo en equipo y lo que es más importante aún, en recursos compartidos. Las universidades van a cumplir un mayor rol no solo a nivel nacional sino, crecientemente, en el desarrollo económico regional, en la implementación del aprendizaje permanente a lo largo de la vida y en el desarrollo de una cultura cívica.

Las universidades serán reconocidas en términos de su conectividad con el sistema de producción de conocimiento socialmente distribuído y su pertinencia determinada por su eficiencia en la consecución de recursos de este sistema. Aunque conserven su papel de "conciencia de la sociedad", la función crítica de las universidades ha sido desplazada a favor de un rol mucho más pragmático en términos de la provisión de talento humano calificado y la producción de conocimiento.

La universidad debe recuperar su protagonismo como actor fundamental y potenciador del sistema social de innovación y por lo tanto debemos estudiar su estructura interna y sus interrelaciones con el entorno.

"La difusión de la producción de conocimiento y de los diferentes contextos de aplicación o su uso en una amplia gama de lugares potenciales genera una explosión en el número de interconexiones, teniendo como resultado una red cuyos nódulos se extienden ahora por todo el globo y cuya conectividad crece a cada dia que pasa". ${ }^{10}$

La nueva forma de producción de conocimiento Modo 2

[...] se lleva a cabo en el contexto de aplicación, caracterizado por: transdisciplinariedad, heterogeneidad, heterarquí y transitoriedad organizativa, responsabilidad social, reflexividad y control de calidad[...]

10 Michael Gibbons, The New production of knowledge: Science and Research in comtemporary societies,( Barcelona:Ediciones Pomares,1997). 
resalta la dependencia del contexto y del uso, resultado de la expansión paralela de los productores y usuarios del conocimiento en la sociedad, institucionalizado en un sistema de producción socialmente distribuido, mas heterogéneo y flexible, como respuesta a las necesidades tanto de la ciencia como de la sociedad. ${ }^{11}$

El Modo 2 interactúa con el llamado Modo 1:

El complejo de ideas, métodos, valores y normas que ha crecido hasta controlar la difusión del modelo newtoniano de ciencia a mas y mas campos de investigación, asegurándose la conformidad con lo que se considera como una práctica científica sana, característico de la investigación disciplinar ampliamente institucionalizado en las universidades. ${ }^{12}$

Conviene identificar con Gibbons cuatro rasgos distintivos en la transdisciplinariedad:

1. Desarrolla un marco bien delimitado, pero en evolución, el cual sirve de guia en la solución de un problema. Esta, dentro del contexto de aplicación, no surge única ni principalmente de la aplicación de un conocimiento que ya existe, pues si bien se deben haber utilizado elementos del conocimiento existente, ha de haber creatividad genuina y el consenso teórico, una vez logrado, no puede reducirse fácilmente a partes de las disciplinas.

2. La solución comprende componentes tanto teóricos como empíricos y aunque, claro está, es una contribución al conocimiento, no necesariamente al conocimiento de una disciplina.

3. Mientras que en el Modo 1 los resultados son comunicados por los canales institucionales, en el Modo 2 se comunican a los que han participado y a medida que lo hacen. Después la difusión se produce principalmente cuando los ejecutantes originales pasan a nuevos contextos de problemas y no por medio de publicaciones científicas o congresos profesionales. La comunicación se desarrolla con base en canales formales e informales. 
4. Es dinámica, pues es la capacidad para resolver problemas en movimiento. Una solución particular puede transformarse en el elemento cognitivo desde el cual se puede seguir desarrollando conocimiento.

Veamos en síntesis las diferencias entre los dos modos.

\begin{tabular}{|c|c|}
\hline Modo 1 & Modo 2 \\
\hline $\begin{array}{l}\text { - Los problemas se plantean y so- } \\
\text { lucionan en el contexto regido } \\
\text { por los intereses principalmente } \\
\text { académicos de una comunidad } \\
\text { específica }\end{array}$ & $\begin{array}{l}\text { - El conocimiento se produce en un } \\
\text { contexto de aplicación }\end{array}$ \\
\hline - Es disciplinar & - Es transdisciplinario \\
\hline $\begin{array}{l}\text { - Se caracteriza por una relativa } \\
\text { homogeneidad }\end{array}$ & - Se caracteriza por su heterogeneidad \\
\hline $\begin{array}{l}\text { - En términos de organización es } \\
\text { jerárquico y en la vida académica } \\
\text { ha tendido a preservar su forma }\end{array}$ & $\begin{array}{l}\text { - Se inclina por una jerarquía más } \\
\text { plana, que se apoya en estructuras } \\
\text { organizacionales transitorias }\end{array}$ \\
\hline $\begin{array}{l}\text { - Responsabilidad eminentemente } \\
\text { académica }\end{array}$ & $\begin{array}{ll}\text { - } & \text { Responsabilidad social y reflexiva } \\
\text { mayor }\end{array}$ \\
\hline $\begin{array}{l}\text { - Evaluación eminentemente co- } \\
\text { legiada }\end{array}$ & $\begin{array}{l}\text { - Prosigue la evaluación colegiada } \\
\text { pero incluye un conjunto de eje- } \\
\text { cutantes más amplio, temporario y } \\
\text { heterogéneo que colaboran en un } \\
\text { problema definido en un contexto } \\
\text { específico y localizado }\end{array}$ \\
\hline $\begin{array}{l}\text { - Las universidades siguen siendo ins- } \\
\text { titucionesrelativamente cerradas }\end{array}$ & $\begin{array}{l}\text { - Las universidades tienen que con- } \\
\text { vertirse en instituciones porosas } \\
\text { más abiertas y dinámicas, en la bús- } \\
\text { queda de alianzas y asociaciones } \\
\text { - La decisión clave de las universi- } \\
\text { dades serála de identificar vincula- } \\
\text { ciones que les permitirán aportar } \\
\text { una contribución eficaz por medio } \\
\text { del uso común de los recursos }\end{array}$ \\
\hline $\begin{array}{l}\text { - Producción de conocimiento } \\
\text { como una actividad relativa- } \\
\text { mente autónoma }\end{array}$ & $\begin{array}{l}\text { - Se evidencia un cambio funda- } \\
\text { mental en tantoque la producción } \\
\text { de conocimiento es una actividad } \\
\text { cada vez menos autónoma }\end{array}$ \\
\hline
\end{tabular}

Fuente: elaboración propia con base en Michael Gibbons en Pertinencia de la Educación Superior en el siglo XXI. 


\section{Redes, universidad y redes universitarias: oportunidad de recu- perar el protagonismo como conciencia crítica por parte de la universidad}

Un componente fundamental de esta indagación está referido a la comprensión del concepto de redes. En esta línea interesa partir de la visión planteada por Fritjof Capra en su propósito de extender al ámbito social, la comprensión de la vida que ha surgido de la teoría de la complejidad.

La nueva comprensión de la vida, basada en los conceptos de la dinámica no lineal, representa un verdadero punto de inflexión conceptual. Disponemos, por primera vez, de un lenguaje eficaz para describir y analizar los sistemas complejos [...].Nuestras disciplinas académicas han sido organizadas de tal modo que las ciencias naturales tratan de estructuras materiales, mientras que las ciencias sociales tratan de las estructuras sociales, que se conciben fundamentalmente como reglas de comportamiento. ${ }^{13}$

Desde esta comprensión y visión de la vida, interesa concebir las redes, desde el planteamiento sistémico, como un patrón común a todo lo vivo. Donde hay vida hay redes. Este emparentamiento de lo biológico con lo social, permite -de entrada- apostarle a una visión de las redes en la que

"la función de cada componente de la red consiste en transformar o sustituír a los demás, de modo que la red entera se genera a si misma de manera continua.Esta es la clave de la definición sistémica de la vida: las redes vivas se crean y se recrean a si mismas sin cesar, mediante la transformación o la sustitución de sus componentes.....al mismo tiempo que mantienen sus patrones de organización en forma de red". ${ }^{14}$

Interesa proyectar la comprensión de las redes con la apropiación de conceptos como la autogénesis, identificada como una característica clave de la vida que los biólogos Humberto Maturana y Francisco Varela calificaron de autopoiésis: que se hace a sí misma. La definición de un sistema vivo como una red autopiésica implica que el fenómeno de la vida tiene que ser considerado una propiedad del sistema como un todo. Premisa conceptual que propicia un abordamiento bien sugerente para el estudio y análisis de las

13 FritjofCapra, Las conexionesocultas,(Barcelona: Editorial Anagrama, 2002), 21 y 22

14 Fritjof Capra, Las conexiones ocultas, 33 . 
redes universitarias regionales de innovación, que responde plenamente a la problemática que se ha propuesto dilucidar.

Este reconocimiento nos exige identificar en cada región, bajo el marco de cada cultura y el particular contexto social en el que busquemos la innovación, reconocer que esta no necesariamente tiene que concebirse como resultado del último desarrollo tecnológico o apareciendo tras correr la frontera del conocimiento en determinado sector o actividad, sino que será de acuerdo con las particulares relaciones sociales y características del territorio que el rescate, aprovechamiento y aplicación de saberes y tradiciones pueden constituir una pertinente y apropiada innovación, en la medida que se traduzca en el fortalecimiento y mejora de las condiciones de la región en la búsqueda del bienestar de sus habitantes.

La innovación no es un fenómeno que emerja única, aislada e independientemente en la producción. Es fundamental hacia el desarrollo de los procesos de innovación el papel de la producción y gestión del conocimiento. Valga precisar, sin embargo, que la identificación de focos individuales, no necesariamente conectados a las redes sociales, regionales, de manera explícita, pueden constituir fuente valiosa de innovación, que necesariamente deberá ser recogida dentro del inventario y la red de conectividad, que propicie su vinculación explícita a los planes que, como en este caso, tratan de la perspectiva y la prospectiva de los distintos antecedentes y focos de innovación hacia el bienestar regional.

Hacia la innovación, las redes ayudan a inducir y difundir los cambios tecnológicos y facilitan el traspaso de conocimientos y experiencias acumuladas tácitamente (knowhow) entre los actores participantes. ${ }^{15}$ Redes que emergen y se proyectan por la interacción, con diversos grados y alcances, de los distintos actores que constituyen el tejido social, bajo las relaciones predominantes y en un estrecho encadenamiento entre los distintos niveles: local, regional, nacional y transnacional.

En la época histórica actual el conocimiento se crea, se transfiere, se aplica, dentro de una interacción cada vez más intensa entre los distintos actores sociales: academia, sector público, sector productivo y sociedad civil.

15 Cecilia Montero y Pablo Morris, Territorio, competitividadsistémica y desarrolloendógeno.Instituciones y actoresdeldesarrollo territorial en el marco de la globalización,(Chile: Ediciones Universidad del Bio Bio- ILPES, 1999), 345. 
Relación que además de multiplicarse e intensificarse, se ha convertido en una expresión de aprendizaje social que propicia el desarrollo de las regiones y países.

La naturaleza, características y condiciones en que se desarrollan las redes de innovación en el mundo contemporáneo permiten afirmar que no se dan solamente por la interacción de factores de carácter tecnológico. La comunicación, la educación, la comunicación educativa son elementos determinantes para la incubación y proyección de los procesos de innovación. Desde la visión de futuro, de las aspiraciones regionales, es fundamental expresar con claridad la estrecha interconexión entre educación, investigación e innovación, como un proceso único e indiviso, dentro del cual la educación y la investigación se conciben unidas orgánicamente hacia su desemboque transformador de las condiciones de existencia en innovación social como fuente de creación y desarrollo de bienestar en cualquiera de los componentes de la vida en la región.

Teórica y metodológicamente se rompe con la delimitación de la innovación al campo económico y se formula una caracterización que involucra los aspectos técnicos o atinentes al desarrollo de estrategias productivas y comerciales, elevándolos hacia un nivel de complejidad en el que las nuevas ideas, los nuevos proyectos, los nuevos procesos, adquieren el estatus de innovación si efectivamente están produciendo una dinámica transformadora de la realidad en un área del conocimiento o de la actividad humana.

El proceso de innovación pasa a ser un proceso de aprendizaje interactivo donde el recurso económico más importante es el conocimiento. El intercambio de este conocimiento es también interactivo, entre diversos actores sociales, lo cual lo hace un fenómeno social. En última instancia, Este fenómeno solo puede ser visto en un contex to territorial determinado caracterizado por su historia, cultura, organización social e institucional. ${ }^{16}$

El concepto de innovación ha ido madurando hacia su caracterización como un proceso de aprendizaje y de creación, de tener un énfasis preponderante en el cambio tecnológico de los procesos productivos, ha accedido hacia

16 Lundvall, Bengt-AkeLundvall, National Systems of Innovation. Towards a theory of Innovation and Interactive Learning, (London: Pinter Publishers, 1997). 
la concepción amplia de innovación social como instrumento no solo del desarrollo empresarial, sino como uno de los componentes centrales para la construcción de opciones de desarrollo de los países.

La dimensión social de la innovación conlleva la visión de la necesidad de creación de un marco interinstitucional que propicie la participación de los diferentes actores sociales en un sistema integral de ciencia, tecnología e innovación.No se trata del hallazgo de una condición emergente en la sociedad contemporánea, sino del proceso de develación de una trama compleja de interacciones. Este proceso de integración de los actores sociales, contando con el papel dinamizador del gobierno, es particularmente importante para los países y regiones atrasadas, promoviendo políticas y programas hacia el desarrollo de la capacidad científica, tecnológica y de innovación. ${ }^{17}$

Los sistemas de innovación surgen entonces con carácter interdisciplinario, involucrando factores económicos, culturales, institucionales, sociales, políticos. No se pueden definir límites o aspectos que no estén involucrados en una visión integral de la dinámica de innovación, concebida dentro de un sistema complejo de interacciones. Cualquier aspecto, expresado desde cualquiera de los actores sociales, tiene su carga de incidencia e impacto sobre la emergencia de la innovación.El desafío conceptual y práctico consiste en hacerlo explícito y consciente en el plan de los actores, en su compromiso con el desarrollo del sistema de innovación.

17 CarlotaPérez, Cambio Técnico, reestructuración competitiva y reforma institucional en los países en desarrollo,(Washington: Banco Mundial. Departamento de análisis estratégico, 1990). 


\section{CONCLUSIÓN}

El estudio de las Redes Universitarias Regionales de Innovación en Colombia permite la identificación de su génesis, la comprensión de su estructura, su funcionamiento y la proyección de estas en el marco global dentro del cual se genera el conocimiento en la época actual. Entendiendo la configuración de las redes como uno de los cauces fundamentales, en la contemporaneidad, de la dinámica y desarrollo educativo.

Emergen redes y se proyectan por la interacción, en diversos grados y alcances, de los actores que constituyen el tejido social bajo el contexto global. El conocimiento se crea, se transfiere y se aplica como consecuencia de un encadenamiento cada vez más estrecho e interactivo entre la academia, el sector público, el sector productivo y la sociedad civil.

Esta visión del rescate esencial de la educación en su más profundo sentido humanista constituye la base para concebir ese vínculo entre conocimiento y educación dentro de una dinámica innovadora. La educación no puede constituir un simple aparato de fortalecimiento del sistema productivo, se abre paso la sustitución del paradigma de la competitividad por el de la cooperación.

El reto está en transformar la información -que se genera por medio de los distintos medios de comunicación-en componente al servicio del aprendizaje social.

Como convertir información en conocimiento útil y como aprovechar el proceso de generación y apropiación del conocimiento para inducir procesos de aprendizaje social, por medio de los cuales el conocimiento crea o fortalece capacidades $y$ habilidades en las personas $u$ organizaciones que se lo apropian. ${ }^{18}$

Sobre el concepto de aprendizaje social conectamos el concepto de red de innovación que vincula en su interacción a actores sociales de muy diversas características.

18 ilbid., 12 
"El consenso social y la concertación política surgiría en una región con el desarrollo de la sinergia cognitiva[...] como una energía colectiva derivada de una interpretación compartida acerca del pasado, el presente y futuro de una región mediante el uso del concepto de conversaciones sociales estructuradas, propio de la lingüística contemporánea[... $]^{\prime \prime}{ }^{19}$

Las redes son un soporte básico de los sistemas regionales de innovación. La forma en que se constituyen, su implantación en el territorio y el tipo de estructura que adopten tienen efectos decisivos en el logro de las metas para sus integrantes. Las redes más virtuosas pueden disparar la dinamización del proceso innovativo y operar como focos de desarrollo.

Estamos, pues ante los retos de la comunicación educativa frente a este nuevo escenario:

Los procesos de comunicación ocupan cada día un lugar más estratégico en nuestra sociedad, puesto que con la información-materia prima, se ubican ya en el espacio de la producción y no solo en el de la circulación. Pero el estudio de esos procesos se halla aún preso de una dispersión disciplinar y metodológica tal que nos hace imposible saber con objetividad qué es lo que ahí está pasando. Estamos entonces urgidos de una teoría capaz de ordenar el campo y delimitar los objetos. ${ }^{20}$

El desarrollo de redes regionales de innovación como nuevo escenario de aprendizaje social es un compromiso y un reto para la universidad como actora del desarrollo, tras la recuperación del protagonismo perdido, donde lo haya tenido. La educación, la pedagogía, la didáctica, el currículo, tendrían que dar su particular contribución al estudio, comprensión y proyección del aprendizaje social, nueva dimensión del aula en el escenario regional. Teniendo como punto de partida que este proceso es el que se desencadena en la emergencia de las redes sociales como mecanismo de comunicación educativa hacia la innovación.

La comunicación, descubierta en esta especie de admiración abrumado$\mathrm{ra}$, semejante a la de los orígenes de la filosofía y la ciencia, no es solo un

19 SergioBoisier, Conversacionessociales y desarrollo regional, (Chile:Universidad de Talca,2000).

20 Jesús Martín Barbero, De los medios a las mediaciones: Comunicación, cultura y hegemonía, (Barcelona: Ediciones G. Gili, 1987),222. 
mero dispositivo para la coordinación de las acciones de diversas personas, sino el acontecer mediante el cual los particulares se abren a los demás y tienen aquella experiencia en la que surge el auténtico compromiso valorativo del hombre como ser genérico y como ciudadano del mundo. ${ }^{21}$

El concepto de aprendizaje social, vinculado al de innovación en el campo educativo, nos conduce inevitablemente al terreno de la construcción de redes de innovación y entre estas las redes universitarias regionales de innovación. Conectando y promoviendo la interacción con todos los actores sociales academia, sector público, sector productivo, sociedad civil- para desplegar un sistema de aprendizaje social, de redes inteligentes, que aprenden y en las que el ingrediente fundamental para el desarrollo de las relaciones de enseñanza-aprendizaje es la cooperación no la competitividad.

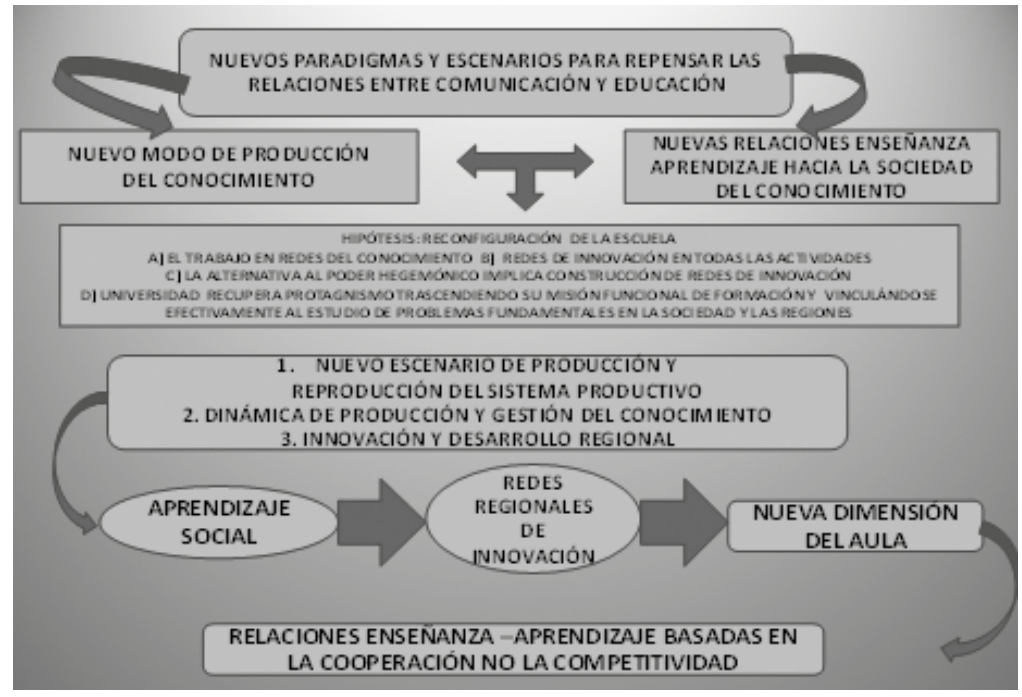

Fuente:elaboración propia.

\section{FUENTES}

Documentos consultados en la Universidad Tecnológica de Pereira: Planes de Desarrollo.

Documentos de la Red de Universidades Estatales de Colombia,Rudecolombia.

Documentos de la Red de Universidades Públicas del Eje Cafetero,Alma Máter.

Vega González, Felipe. “Emergencia de la función de Innovación en la Universidad: El proceso en la Universidad Tecnológica de Pereira" (Tesis de Maestría, Universidad Tecnològica de Pereira, , 2010).

21 GuillermoHoyosVásquez, Comunicación, educación y ciudadanía,(Bogotá, Siglodel Hombre editores, 2007), 82. 


\section{REFERENCIAS}

Barbero, Jesús Martín.De los medios a las mediaciones: Comunicación, cultura y hegemonía.Barcelona: Ediciones G. Gili, 1987.

Bengt-AkeLundvall.National Systems of Innovation.Towards a theory of Innovation and Interactive Learning. London: Pinter Publishers, 1997.

Boisier, Sergio.Conversaciones sociales y desarrollo regional. Chile: Universidad de Talca,2000.

Boisier, Sergio.Un ensayo epistemológico y axiológico sobre gestión del desarrollo territorial: conocimiento y valores. Santiago de Chile: Cepal, 2005.

Capra, Fritjof. Las conexiones ocultas.Barcelona: Editorial Anagrama, 2002.

Chaparro Osorio, Fernando. "De las sociedades de la información a las sociedades del conocimiento". En Conocimiento, innovación y construcción de sociedad. Editado por TercerMundoeditores, 1998.

Gibbons, Michael. The New production of knowledge: Science and Research in comtemporary societies. Barcelona:EdicionesPomares, 1997.

HoyosVásquez, Guillermo. Comunicación, educación y ciudadanía. Bogotá:Siglo del Hombre editores, 2007.

Immanuel Wallerstein. El legado de la sociología, la promesa de la ciencia social. Monteral: XXIV Congreso Mundial de Sociología, 1998.

Mejìa Jaramillo, Marco Raúl. Educacion(es) en la(s) globalización(es). Entre el pensamiento único y la nueva crítica. Bogotá: Ediciones desde abajo, 2006.

Montero, Cecilia y Pablo Morris.Territorio, competitividad sistémica y desarrollo endógeno. Instituciones y actores del desarrollo territorial en el marco de la globalización. Chile: Ediciones Universidad del Bio Bio- ILPES, 1999.

Morin, Edgar. "Principios del conocimiento pertinente - Reducción y disyunción". En Los siete saberes necesarios para la educación del futuro, Ediciones Unesco Paris-Francia, 1999.

Pérez, Carlota. Cambio Técnico, reestructuración competitiva y reforma institucional en los países en desarrollo.Washington: Banco Mundial. Departamento de análisis estratégico, 1990.

UNESCO, Consejo del Informe Mundial. Hacia las sociedades del conocimiento.Paris: Ediciones Unesco, PDF en Internet, 2005.

\begin{tabular}{|l|}
\hline $\begin{array}{l}\text { Vega González, Felipe. "Redes universitarias regionales de innovación nuevo } \\
\text { escenario de aprendizaje social”. Revista Historia de la Educación Latinoameri- } \\
\text { cana. Vol. } 16 \text { No, 22, (2014): } 213 \text { - } 229 .\end{array}$ \\
\hline
\end{tabular}

